\title{
Alain Boillat, En cas de malheur de Simenon à Autant-Lara. Essai de génétique scénaristique, Genève, Droz, 2020, 376 p.
}

\section{Olivier Lumbroso}

\section{(2) OpenEdition Journals}

Édition électronique

URL : https://journals.openedition.org/genesis/5928

DOI : $10.4000 /$ genesis.5928

ISSN : 2268-1590

Éditeur :

Presses universitaires de Paris Sorbonne (PUPS), Société internationale de génétique artistique littéraire et scientifique (SIGALES)

\section{Édition imprimée}

Date de publication : 1 juillet 2021

Pagination : 214-216

ISBN : 979-10-231-0710-4

ISSN : $1167-5101$

\section{Référence électronique}

Olivier Lumbroso, «Alain Boillat, En cas de malheur de Simenon à Autant-Lara. Essai de génétique scénaristique, Genève, Droz, 2020, 376 p. », Genesis [En ligne], 52 | 2021, mis en ligne le 01 juillet 2021, consulté le 17 août 2022. URL : http://journals.openedition.org/genesis/5928 ; DOI : https://doi.org/ 10.4000/genesis.5928 
par une pensée libre d'associations, hors de toutes convenances.

Sont concernés aussi bien les architectes que les musiciens, les peintres, les écrivains, les botanistes et même les cuisiniers, tel Ferran Adrià, un des meilleurs chefs au monde. Mais passé cet intérêt particulier, ce volume s'adresse au lecteur universel à qui il montre que la pensée, avant d'être langage verbal, est peut-être comme le disent Nicole Marchand-Zañartu et Jean Lauxerois « dans son frémissement initial, [...] d'emblée image» mais pas seulement.

Ce «frémissement initial» fait appel non pas à ces représentations usuelles : dessin, trait, ou image, mais justement à une projection exempte de codification, une idiosyncrasie qui, pour le peintre, le botaniste, l'architecte, met l'éclair de la pensée pure à distance d'une pensée captive des modes conventionnels de la représentation.

Mais que faire de telles images dans la démarche d'un généticien du texte? L'analyse génétique tente d'observer l'origine, le sillage tracés par une œuvre, son contexte, sa limite.

Si les images de pensées ne sont pas des œuvres, elles participent à leur devenir. «Figure, schème, schéma, dessin, dessin préparatoire, esquisse, croquis...» pour reprendre quelques-uns des vocables utilisés dans la postface de Nicole MarchandZañartu et Marie-Haude Caraës ${ }^{2}$, elles sont tout cela à la fois et tout autre chose. Il faut donc s'attacher à les observer avec un regard neuf, juste observer ce qu'elles diffusent en nous de compréhension de la création car on ne peut pas en rester à cette supposée origine. Il faut la confronter au contexte de sa production.

Deux questions essentielles auxquelles N. Marchand-Zañartu et Jean Lauxerois tentent d'apporter leur éclairage et conduisent le lecteur arrivé au terme de sa consultation et au seuil de la postface de 32 grammes : 1) Ces matériaux sont-ils des images? 2) Qu'est-ce qui nous permet de signifier que ces images seraient «de pensée»?

Jean Lauxerois, spécialiste de la pensée grecque et dont le travail porte entre autres sur le sens de l'œuvre d'art et de la culture, livre dans cette postface une réflexion philosophique sur la production de ces images, convoquant dans sa démonstration «le beau sans schème » de Socrate, «l'imagination au cœur du fonctionnement de la raison » chez Kant, aussi bien que la pensée de Benjamin pour qui «le gramme concrétise l'image de pensée comme telle, laquelle peut même apparaître sous trois modes grammatiques différents, en prenant ainsi plusieurs sens».

32 grammes est plus qu'un livre d'images, c'est une question en abyme, celle de la création avant la création, de l'origine avant une origine supposée, et d'autant plus un livre pour les généticiens que «le gramme n'est pas une œuvre : il est (son) l'amorce, (sa) une greffe initiale». Leçon à méditer !

Alain Boillat, En cas de malheur de Simenon à Autant-Lara. Essai de génétique scénaristique, Genève, Droz, 2020, 376 p.

\section{Compte rendu par Olivier Lumbroso*}

Dans le domaine des études sur l'adaptation cinématographique des œuvres littéraires, l'essai d'Alain Boillat propose une démarche novatrice sur plusieurs plans théoriques et analytiques. Il se fonde, en effet, sur une approche génétique globale des scénarios, manuscrits et tapuscrits d'En cas de malheur (1956) de Georges Simenon. Cet ensemble se voit saisi dans l'enchaînement entrelacé des diverses campagnes d'écriture qui produisent ces écrits préparatoires : "premier incipit», «plans scénariques », «scénarios partiels», «continuités dialoguées» et «scénario de tournage ». Ainsi, sont décrites et interprétées les traces de la genèse scénaristique souvent négligées par les études cinématographiques : la matérialité des supports et des graphies (papier, recto/verso, écritures linéaire/tabulaire, didascalies...), la datation et la temporalité relative (séquentialité, segmentation, montage et rythme, scènes et plans, flash-backs...), les fonctions de régie et de mise en scène (diégèse, décor, éclairage, habillement, ton...), l'évaluation des coûts (production, bobines, studios...), la complexité énonciative de l'écriture collaborative, fréquente dans les pratiques scénaristiques du «cinéma d'équipe», à l'œuvre justement dans le travail des scénaristes d'En cas de malheur, Jean Aurenche et Pierre Bost, travail dans lequel s'immisce précocement le réalisateur luimême, Claude Autant-Lara.

Par cette approche multidimensionnelle qui emprunte à la génétique sa terminologie et sa méthode, l'essai s'écarte autant d'une conception simpliste de l'adaptation comme translation d'un médium vers un autre, que d'une vision seulement formaliste, qui réduirait l'encodage filmique à une traduction sémiotique, ligne à ligne, du langage littéraire du roman. L'adaptation suppose l'altérité : «La recherche "d'équivalences" vilipendée par Truffaut se situe bien au principe de la méthode des adaptateurs, mais on peut dire qu'elle est en fait requise dans toute démarche de transposition ou de traduction visant à conserver le texte premier comme référent principal tout en l'adaptant à un langage, à des intentions de représentations et un public-cible autres » (p. 322). De façon à la fois ambitieuse et instrumentée sur le plan critique, il s'agit d'ouvrir le «chantier scénaristique» du film, sorti le 17 septembre 1958, en soulignant les virtualités, les tâtonnements et les repentirs au cours de la préparation d'un canevas protéiforme qui débute le 8 avril 1957. Le spectacle de la genèse en acte est d'autant plus fascinant qu'il tient compte des voix multiples, convergentes, complémentaires ou divergentes de l'équipe du film, du premier scénario jusqu' au tournage, en studio et dans Paris, du 4 novembre 1957 au 4 février 1958. L'entreprise de dépliement des textes et des voix de la genèse de ce film en particulier échappe aussi à la logique simplificatrice et généraliste du « manuel»,

2. Nicole Marchand-Zañartu et Marie-Haude Caraës, Images de pensée, op. cit. (*) Université Sorbonne Nouvelle-Paris 3. 
où l'extrait, le plus souvent illustratif, sert une forme de normativité des scénariostypes à des fins didactiques. Ici le «chantier» convoque les écrits de travail, le carnet de bord méta-scénaristique du réalisateur, les correspondances, les rencontres, les séances préparatoires, en somme un atlas de matériaux qui donne l'environnement des «variantes scénaristiques».

Composé de façon logique et déductive, l'ouvrage est constitué de six chapitres qui entrecroisent les questions littéraires et cinématographiques, les aspects théoriques et empiriques, les analyses génétiques et les données culturelles qui les situent dans une époque. Les deux premiers chapitres partent de la matière du roman et du film, en abordant les questions centrales de l'adaptation : la «visualité» de l'écriture de Simenon, le traitement de la «sexualité», la rhétorique $\mathrm{du}$ «crescendo» et la construction du «point de vue». Le chapitre III aborde les modalités possibles d'une application de l'hypothèse génétique au scénario filmique. En effet, littérature et cinéma adoptent deux types distincts de processus créatif. Prenons, par exemple, leur cadre énonciatif respectif : le canevas d'un écrivain est un «écrit-poursoi» qui n'engage que l'auteur évaluateur de lui-même, alors que le scénario d'un film est destiné à plusieurs membres de l'équipe qui forment des instances de validation du contenu. Le canevas de l'écrivain prépare une étape d'écriture ultérieure tandis que le scénario filmique est une phase rédactionnelle, à visées pragmatique et économique, qui débouche sur un tournage.

Pourtant, ces différences n'invalident ni la démarche matérialiste et empirique ni la procédure d'établissement du «dossier génétique» ni les quatre opérations fondamentales du raturage. La méthode génétique s'avère heuristique dans le cas présent, autant pour décrire «l'horizon des possibles» du récit filmique et les pratiques scénaristiques, que le rôle des vedettes et le cahier des charges historicisé des représentations genrées. Soulignons que l'essai pose de nombreuses autres questions méthodologiques qui s'avèrent communes aux corpus littéraire et cinématographique : comment aborder la béance qui sépare parfois les «variantes scénaristiques» et l'œuvre lorsqu'il est difficile d'accéder aux «variantes filmiques»? L'étude de la genèse d'une œuvre ne tire-t-elle pas bénéfice à faire dialoguer l'avant-texte et les correspondances, les carnets, les journaux de bord, plus largement les phénomènes d'interdiscursivité au sein d'une génétique culturelle?

Après un état des lieux et des propositions théoriques audacieuses qui ne font pas fi de certaines précautions, les chapitres IV et V immergent le lecteur dans la genèse du scénario à partir des plans scénariques et des canevas partiels qui aboutissent au «scénario de tournage». Ce dernier constitue, certes, une finalisation mais pas un achèvement, dans la mesure où la genèse d'un film est, jusqu'au bout, une affaire collective. Les «stars» aussi infléchissent le sens des personnages, selon des stratégies de représentations en lien avec leur persona médiatique. Ainsi Edwige Feuillère tint à recevoir le scénario trois mois avant le tournage. Jean Gabin entra en conflit avec Claude Autant-Lara, à propos de son personnage, l'avocat André Gobillot, afin que soit mieux soulignée la masculinité positivée d'un «homme authentique». Réciproquement, au fil de la variance scénaristique, le réalisateur et les scénaristes modulent ou amplifient la «centralité » des personnages de premier plan : le couple Bardot/Gabin est ainsi au cœur d'un réglage subtil de leur capital charismatique (sensualité, sincérité, paternalisme, rapports de classes). À titre d'exemple, on peut épingler, au cours de la préparation du scénario, cette autoconsigne d'Autant-Lara, à propos $\mathrm{du}$ «potentiel érotique» de Bardot : la faire jouer «monotone».

L'un des apports les plus neufs de l'essai tient à son positionnement théorique interdisciplinaire : ne pas en rester à une analyse strictement textuelle des reformulations des états du scénario, selon une logique des variantes que les spécialistes littéraires connaissent bien quand ils étudient des manuscrits d'écrivains. Avec l'idée de cerner au plus près les conditions de l'écriture et de ses choix, l'essai croise, avec grand profit, la critique génétique et les cultural studies. Deux domaines sont notamment convoqués : les gender studies, qui interrogent les représentations genrées à l'aune des normes en vigueur dans la société française des années 1950 et les star studies, selon une étude ponctuelle à l'intersection des carrières de deux "mythes" du cinéma, Brigitte Bardot et Jean Gabin, en présence de vedettes de premier plan, Edwige Feuillère et Nicole Berger. Ces deux domaines des études culturelles ouvrent l'empan des déterminations d'un imaginaire collectif qui oriente, sur le plan idéologique des règles et de leurs transgressions, la fabrique du scénario et sa réception, dans un champ culturel sous tension, notamment au seuil de la Nouvelle Vague.

De fait, l'essai décrit non seulement, avec soin, les archives du film d'AutantLara, mais aussi, à partir de cette description méthodique du matériau, en propose des interprétations fines, par exemple les infléchissements liés à la persona des vedettes impliquées dans le casting du film, les effets de l'autocensure sur les scénaristes en lien avec les préconstruits idéologiques d'époque ou encore les choix de «désambigüiser» la romance d'Yvette et Gobillot, de renforcer les moments d'identification et d'empathie, pour la rendre plus accessible à une large audience populaire. Ainsi de la séquence consacrée au meurtre d'Yvette par son amant Mazetti, à la fin du film. Celui-ci s'achève sur Gabin, vu de dos, qui s'éloigne le long du quai de Javel. Précisons que ces éléments situationnels, historicisés et périodisés, ne fournissent pas seulement le «contexte» général, coupé du processus d'écriture. Par une approche de nature sociocritique, l'essai en souligne l'affleurement scriptural sans atténuer les tensions et les résolutions, au cours d'un processus d'absorption que manifestent les réécritures et même les marginalia des textes scénaristiques successifs, profondément dialogiques et dialogués. Il est important de souligner que ce processus dynamique des réécritures est multiscalaire, croisant les échelles et les dimensions, de sorte que les modifications 
apportées aux scénarios, par exemple la linéarisation de la chronologie, ont très souvent des répercussions transversales sur l'ensemble du film.

Cet essai passionnant, copieux par ses notes de bas de page, fera date sans aucun doute car, à la manière d'un pari intellectuel, il trace un sillon nouveau et fonde une méthode. Il ne se réduit pas à une étude de cas, passionnante par ailleurs, à propos d'un film qui méritait ces analyses abordant, selon la perspective des «équivalences », les fidélités autant que les recréations de l'adaptation, depuis la chronologie du film jusqu' au paramétrage du «regard spectatoriel». Avec un souci double de rigueur et d'originalité, il nourrit aussi la réflexion épistémologique sur les périmètres et les objets, les méthodes et les enjeux de la génétique des textes et des arts, dans le domaine des études cinématographiques, raison pour laquelle il serait sûrement passionnant de construire, tel un second pari, un pont réciproque entre cette "génétique scénaristique» du film et la «génétique scénarique » des œuvres littéraires, proposée par Henri Mitterand, lors du colloque Zola. Genèse de l'œuvre, en 1999. Enfin, l'essai ouvre, sans aucun doute, une «perspective inédite sur l'histoire des techniques cinématographiques », celle qui, dans l'avenir, «conjuguerait une histoire esthétique relative à des procédés spécifiques (mouvement d'appareil, trucages, raccords, etc.) et une histoire discursive (l'une et l'autre étant interdépendantes)» (p. 269).

Geneviève Henrot Sostero (dir.), Archéologie(s) de la traduction, Paris, Classiques Garnier, coll. «Translatio », 2020, 303 p.

\section{Compte rendu par Patrick Hersant*}

Aux éditions Classiques Garnier, la collection «Translatio» accueille depuis l'an dernier, en douze volumes thématiques dont la moitié sont encore à paraître, les actes du congrès de traductologie qui s'est tenu à l'université Paris-Nanterre en avril 2017.
Le troisième de la collection, Archéologie(s) de la traduction, est le plus étroitement lié aux préoccupations génétiques - sa directrice scientifique, Geneviève Henrot Sostero, est du reste membre de l'ITEM, comme plusieurs contributeurs du volume. En plus d'un avant-propos et d'une première partie exposant les «Fondements théoriques» des études rassemblées, tous deux signés par Henrot Sostero, l'ouvrage se compose de quatre sections dédiées aux brouillons d'auteurs, à la pensée traductologique, à l'anamnèse et à l'observation du geste traductif lui-même.

L'avant-propos de cette «archéologie» consiste en un précieux état des lieux de la recherche en matière de génétique des traductions depuis une vingtaine d'années. Les premières hypothèses traductologiques se forment autour de grands auteurs pratiquant la traduction, tels Paul Valéry ou Saint-John Perse, et connaissent un écho particulier au Brésil où sont organisés les premiers colloques sur le sujet; d'autres suivront en France, accompagnés ou précédés par la parution de numéros thématiques - la revue brésilienne Manuscrítica en 2011, Genesis en 2014, Linguistica antverpiensia et Transalpina en 2015, Palimpsestes en 2020, Meta en 20213. Paraissent dans ce sillage des travaux qui s'attachent, au moins en partie, à «détecter les traces qui permettront de supputer les opérations de l'esprit sollicitées par l'entreprise, les tactiques et stratégies mises en œuvre, les projets incarnés et portés à l'existence» (p. 11).

Sous l'intitulé «Pour une génétique de la traduction : concepts, méthodes, visées », la première partie défend et illustre sur plus de quarante pages une approche pluraliste conviant les sciences cognitives, les sciences du langage, la traductologie, la philologie et la génétique de la création. Quatre sous-parties abordent successivement (1) la philologie et la génétique, ces «deux sciences du texte envisagé dans son feuilleté diachronique » (p. 18) dont Henrot Sostero souligne les oppositions en matière de «terroir» (manuscrits anciens ou modernes, dont les statuts respectifs sont l'occasion de rappels historiques bienvenus), de démarche et de productions; (2) les acquis de la génétique française, dont Henrot Sostero, dans un long développement qui sera fort utile aux non-spécialistes, passe les concepts et les méthodes en revue afin de les «élargir à la génétique de la traduction », non sans rappeler que le statut second de la traduction et l'auctoritas fragile des traducteurs expliquent la relative minceur d'un corpus où les traducteurs également (ou surtout) écrivains sont surreprésentés ; (3) les apports des sciences du langage, et notamment de certains concepts permettant d'observer l'opération d'écriture - comme l'énonciation, la polyphonie énonciative, la paraphrase, le métalangage et la stylistique - à un stade où abondent encore «les possibles », ou de la question de la textualité de l'avant-texte; (4) les avancées de la traductologie dues aux sciences cognitives, avec par exemple la verbalisation à chaud de l'activité traduisante (think-aloud protocols) et la multiplication de logiciels spécialisés.

Dans sa contribution, Florence Pellegrini applique au dossier génétique de Bouvard et Pécuchet le concept d' «orthonymie», cette tendance qui inciterait tout traducteur à préférer la façon de dire la plus spontanée et la plus usuelle 4 . À partir de cinq traductions italiennes, elle montre que plusieurs traducteurs sont revenus à un état de la genèse que Flaubert avait précisément écarté. Une énonciation ambiguë

(*) Paris 8, ITEM (équipe Multilinguisme, traduction, création).

3. Par ordre chronologique : Manuscrítica, no 20 , 2011 (en ligne sur revistas.usp.br); Genesis, no 38, 2014 (openedition.org); Linguistica antverpiensia, $\mathrm{n}^{\circ} 14$ (lans-tts.uantwerpen.be); Transalpina, no 18, 2015; Palimpsestes, no 34 , 2020 (openedition.org); et Meta, no 65 (2), 2021 (à paraître).

4. Jean-Claude Chevalier a conceptualisé, sous le terme d' «orthonymie » emprunté à Bernard Pottier, un phénomène de normalisation lexicale, sorte de degré zéro de la diction visant l'expression directe et naturelle dans une formulation «toute nue, toute droite, toute simple». Jean-Claude Chevalier et Marie-France Delport, L'Horlogerie de saint Jérôme : problèmes linguistiques de la traduction, Paris, L'Harmattan, 1995. 